\title{
Influence of Initial Stress and Gravity Field on Propagation of Rayleigh and Stoneley Waves in a Thermoelastic Orthotropic Granular Medium
}

\author{
S. M. Ahmed ${ }^{1}$ and S. M. Abo-Dahab ${ }^{2,3}$ \\ ${ }^{1}$ Department of Mathematics, Faculty of Education, Suez Canal University, EL-Arish 45111, Egypt \\ ${ }^{2}$ Department of Mathematics, Faculty of Science, Taif University, Taif, Saudi Arabia \\ ${ }^{3}$ Department of Mathematics, Faculty of Science, South Valley University, Qena 83523, Egypt
}

Correspondence should be addressed to S. M. Abo-Dahab, sdahb@yahoo.com

Received 9 May 2011; Revised 11 October 2011; Accepted 26 October 2011

Academic Editor: Moran Wang

Copyright (C) 2012 S. M. Ahmed and S. M. Abo-Dahab. This is an open access article distributed under the Creative Commons Attribution License, which permits unrestricted use, distribution, and reproduction in any medium, provided the original work is properly cited.

The propagation of Rayleigh and Stoneley waves in a thermoelastic orthotropic granular half-space supporting a different layer under the influence of initial stress and gravity field is studied. The frequency equation of Rayleigh waves in the form of twelfth-order determinantal expression and the frequency equation of Stoneley waves in the form of eighth-order determinantal expression are obtained. The standard equation of dispersion is discussed to obtain Rayleigh and Stoneley waves that have complex roots; the real part gives the velocity of Rayleigh or Stoneley waves but the imaginary part gives the attenuation coefficient. Finally, the numerical results have been given and illustrated graphically, and their physical meaning has been explained.

\section{Introduction}

The propagation of thermoelastic waves in a granular medium under initial stress and gravity field has applications in soil mechanics, earthquake science, geophysics, mining engineering, and so forth. The theoretical outline of the development of the subject from the mid-thirties was given by Paria [1]. The present paper, however, is based on the dynamics of granular media as propounded by Oshima [2,3]. The medium under consideration is discontinuous such as one composed numerous large or small grains. Unlike a continuous body, each element or grain cannot only translate but also rotate about its centre of gravity. This motion is the characteristics of the medium and has an important effect upon the equation of motion to produce internal friction. It is assumed that the medium contains so many grains that they will never be separated from each other during the deformation, and that the grain 
has perfect elasticity. The propagation of Rayleigh waves in granular medium was given by many authors such as Bhattacharyya [4], El-Naggar [5], Ahmed [6, 7], and others. Ahmed [8] discussed Stoneley waves in a nonhomogeneous granular medium under the influence of gravity.

The problem of Stoneley waves plays an important role in the earthquake science, optics, geophysics, and plasma physics. Many authors such as Abd-Alla and Ahmed [9, 10], El-Naggar et al. [11], Das et al. [12], and others investigated the effect of gravity of the propagation of surface waves (Stoneley waves and Rayleigh waves) in an elastic solid medium. Goda [13] illustrated the effect of inhomogeneity and anisotropy on Stoneley waves; Abd-Alla et al. [14] discussed Rayleigh waves in a magnetoelastic half-space of orthotropic material under influence of initial stress and gravity field. Sharma et al. [15] studied the propagation characteristics of Rayleigh waves in transversely isotropic piezothermoelastic half-space with rotation and thermal relaxation. Sharma et al. [16] investigated the problem of propagation characteristics of Rayleigh waves in transversely isotropic piezothermoelastic materials. Sharma and Walia [17] investigated Rayleigh waves in piezothermoelastic materials. Ting [18] illustrated the surface waves in a rotating anisotropic elastic half-space. Abd-Alla and Abo-Dahab [19] investigated Rayleigh waves in magneto-thermo-viscoelastic solid with thermal relaxation times. Recently, Ahmed and Abo-Dahab [20] pointed out Propagation of Love waves in an orthotropic granular layer under initial stress overlying a semi-infinite granular medium. Vinh and Seriani [21] illustrated explicit secular equations of Rayleigh waves in a nonhomogeneous orthotropic elastic medium under the influence of gravity. Vinh and Seriani [22] discussed the explicit secular equations of Stoneley waves in a nonhomogeneous orthotropic elastic medium under the influence of gravity. Vinh [23] discussed the explicit secular equations of Rayleigh waves in elastic media under the influence of gravity and initial stress. Lo [24] investigated the propagation and attenuation of Rayleigh waves in a semi-infinite unsaturated poroelastic medium. El-Naggar [25] investigated the dynamical problem of a generalized thermoelastic granular infinite cylinder under initial stress. Recently, Abd-Alla et al. [26] investigated Rayleigh waves in generalized magneto-thermo-viscoelastic granular medium under the influence of rotation, gravity field.

This paper is devoted to study the effect of gravity field and the initial stress on the propagation of Rayleigh and Stoneley waves in thermoelastic orthotropic granular halfspace supporting a different layer under initial stress and gravity field. The frequency equations are obtained: the frequency equation of Rayleigh waves in the form of twelfth-order determinantal expression and the frequency equation of Stoneley waves in the form of eighthorder determinantal expression. The standard equation of dispersion is discussed to obtain the Rayleigh and Stoneley waves that have complex roots; the real part gives the velocity of Rayleigh or Stoneley waves but the imaginary part gives the attenuation coefficient. The results obtained are displayed graphically and their physical meaning has been explained.

\section{Formulation of the Problem}

Let us consider an initially stressed orthotropic granular layer of finite thickness $H$ overlaying a semi-infinite orthotropic granular medium. The upper surface of the upper layer is assumed to be free and horizontal. We take a set of orthogonal Cartesian axes $O x_{1} x_{2} x_{3}$ such that the interface and the free surface of the granular layer resting on the granular half-space of different material are the planes $x_{3}=H$ and $x_{3}=0$, respectively, with the origin $O$ being any point on the interface surface; $x_{3}$-axis is positive in the direction towards the exterior of the 
half-space, and $x_{1}$-axis is positive along the direction of Rayleigh waves and Stoneley waves propagation. Let the both media be under initial compression stress $P$ along $x_{1}$-axis, with the influence of gravity and at initial temperature $T_{0}$. It is assumed that both media exchange heat freely with their surroundings; an initial stress is produced by a slow process of creep, where the shear stresses tend to become small or vanish after a long interval of time.

In view of the two-dimensional nature of the problem, we assume that the state of initial stress is

$$
\tau_{11}=\tau_{33}=\tau, \quad \tau_{13}=0,
$$

the equilibrium conditions of the initial stress field are given by [12]

$$
\frac{\partial \tau}{\partial x_{1}}=0, \quad \frac{\partial \tau}{\partial x_{3}}-\rho g=0
$$

The state of deformation in the granular medium is described by the displacement vector $\vec{U}=\left(u_{1}, 0, u_{3}\right)$ of the centre of gravity of a grain and the rotation vector $\vec{\xi}=(\xi, \eta, \zeta)$ of the grain about its centre of gravity. There exist a stress tensor and a couple stress which are nonsymmetric, that is,

$$
\tau_{i j} \neq \tau_{j i}, \quad M_{i j} \neq M_{j i} \quad(i, j=1,2,3) .
$$

The stress tensor $\tau_{i j}$ can be expressed as the sum of symmetric and antisymmetric tensors

$$
\tau_{i j}=\sigma_{i j}+\sigma_{i j}^{\prime}
$$

where

$$
\sigma_{i j}=\frac{1}{2}\left(\tau_{i j}+\tau_{j i}\right), \quad \sigma_{i j}^{\prime}=\frac{1}{2}\left(\tau_{i j}-\tau_{j i}\right)
$$

The symmetric tensor $\sigma_{i j}=\sigma_{j i}$ is related to the symmetric strain tensor

$$
e_{i j}=e_{j i}=\frac{1}{2}\left(\frac{\partial u_{i}}{\partial x_{j}}+\frac{\partial u_{j}}{\partial x_{i}}\right)
$$

by the Hook's law.

The antisymmetric stress $\sigma_{i j}^{\prime}$ is given by

$$
\sigma_{23}^{\prime}=-F \frac{\partial \xi}{\partial t}, \quad \sigma_{31}^{\prime}=-F \frac{\partial \eta}{\partial t}, \quad \sigma_{12}^{\prime}=-F \frac{\partial \xi}{\partial t}, \quad \sigma_{11}^{\prime}=\sigma_{22}^{\prime}=\sigma_{33}^{\prime}=0
$$


the couple stress $M_{i j}$ is given by

$$
\begin{gathered}
M_{i j}=M v_{i j}, \\
v_{11}=\frac{\partial \xi}{\partial x_{1}}, \quad v_{22}=0, \quad v_{33}=\frac{\partial \zeta}{\partial x_{3}}, \quad v_{23}=0, \\
v_{31}=\frac{\partial \xi}{\partial x_{3}}, \quad v_{12}=\frac{\partial}{\partial x_{1}}\left(\eta+\omega_{2}\right), \quad v_{32}=\frac{\partial}{\partial x_{3}}\left(\eta+\omega_{2}\right), \\
v_{13}=\frac{\partial \zeta}{\partial x_{1}}, \quad v_{21}=0,
\end{gathered}
$$

where, $\omega_{2}=\partial u_{1} / \partial x_{3}-\partial u_{3} / \partial x_{1}$.

The six equations of motion are $[9,11,12]$

$$
\begin{gathered}
\frac{\partial \tau_{11}}{\partial x_{1}}+\frac{\partial \tau_{31}}{\partial x_{3}}-\frac{P}{2} \frac{\partial \omega_{2}}{\partial x_{3}}-\rho g \frac{\partial u_{3}}{\partial x_{1}}=\rho \frac{\partial^{2} u_{1}}{\partial t^{2}} \\
\frac{\partial \tau_{12}}{\partial x_{1}}+\frac{\partial \tau_{32}}{\partial x_{3}}=0 \\
\frac{\partial \tau_{13}}{\partial x_{1}}+\frac{\partial \tau_{33}}{\partial x_{3}}-\frac{P}{2} \frac{\partial \omega_{2}}{\partial x_{1}}+\rho g \frac{\partial u_{1}}{\partial x_{1}}=\rho \frac{\partial^{2} u_{3}}{\partial t^{2}} \\
\tau_{23}-\tau_{32}+\frac{\partial M_{11}}{\partial x_{1}}+\frac{\partial M_{31}}{\partial x_{3}}=0 \\
\tau_{31}-\tau_{13}+\frac{\partial M_{12}}{\partial x_{1}}+\frac{\partial M_{32}}{\partial x_{3}}=0 \\
\tau_{12}-\tau_{21}+\frac{\partial M_{13}}{\partial x_{1}}+\frac{\partial M_{33}}{\partial x_{3}}=0 .
\end{gathered}
$$

The components of stress for orthotropic body, under the effect of an initial compression stress $P$, are given by [11]

$$
\begin{gathered}
\tau_{11}=\left(c_{11}+P\right) \frac{\partial u_{1}}{\partial x_{1}}+\left(c_{13}+P\right) \frac{\partial u_{3}}{\partial x_{3}}-v_{1} T, \quad \tau_{33}=c_{13} \frac{\partial u_{1}}{\partial x_{1}}+c_{33} \frac{\partial u_{3}}{\partial x_{3}}-v_{3} T \\
\tau_{31}=c_{55}\left(\frac{\partial u_{1}}{\partial x_{3}}+\frac{\partial u_{3}}{\partial x_{1}}\right)-F \frac{\partial \eta}{\partial t}, \quad \tau_{13}=c_{55}\left(\frac{\partial u_{1}}{\partial x_{3}}+\frac{\partial u_{3}}{\partial x_{1}}\right)+F \frac{\partial \eta}{\partial t}
\end{gathered}
$$

where

$$
v_{1}=\left(c_{11}+c_{12}\right) \alpha_{1}+c_{13} \alpha_{2}, \quad v_{3}=2 c_{13} \alpha_{1}+c_{33} \alpha_{2} .
$$


Substituting (2.9) into (2.10), we obtain

$$
\begin{aligned}
& \left(c_{11}+P\right) \frac{\partial^{2} u_{1}}{\partial x_{1}^{2}}+\left(c_{55}+\frac{P}{2}\right) \frac{\partial^{2} u_{1}}{\partial x_{3}^{2}}+\left(c_{13}+c_{55}+\frac{P}{2}\right) \frac{\partial^{2} u_{3}}{\partial x_{1} \partial x_{3}}-\frac{\partial}{\partial x_{1}}\left(v_{1} T\right)-\rho g \frac{\partial u_{3}}{\partial x_{1}} \\
& -F \frac{\partial}{\partial t}\left(\frac{\partial \eta}{\partial x_{3}}\right)=\rho \frac{\partial^{2} u_{1}}{\partial t^{2}} \\
& F \frac{\partial}{\partial t}\left(\frac{\partial \xi}{\partial x_{3}}-\frac{\partial \zeta}{\partial x_{1}}\right)=0 \\
& \left(c_{55}+c_{13}+\frac{P}{2}\right) \frac{\partial^{2} u_{1}}{\partial x_{1} \partial x_{3}}+\left(c_{55}-\frac{P}{2}\right) \frac{\partial^{2} u_{3}}{\partial x_{1}^{2}}+c_{33} \frac{\partial^{2} u_{3}}{\partial x_{3}^{2}}-\frac{\partial}{\partial x_{3}}\left(v_{3} T\right)+\rho g \frac{\partial u_{1}}{\partial x_{1}} \\
& +F \frac{\partial}{\partial t}\left(\frac{\partial \eta}{\partial x_{1}}\right)=\rho \frac{\partial^{2} u_{3}}{\partial t^{2}}, \\
& -2 F \frac{\partial \xi}{\partial t}+\nabla^{2}(M \xi)=0, \quad-2 F \frac{\partial \eta}{\partial t}+\nabla^{2}\left[M\left(\eta+\frac{\partial u_{1}}{\partial x_{3}}-\frac{\partial u_{3}}{\partial x_{1}}\right)\right]=0 \\
& -2 F \frac{\partial \zeta}{\partial t}+\nabla^{2}(M \zeta)=0
\end{aligned}
$$

The heat conduction equation is given by [11]

$$
\nabla^{2} T-\frac{1}{x} \frac{\partial T}{\partial t}-\varepsilon \nabla \cdot\left(\frac{\partial \vec{U}}{\partial t}\right)=0
$$

where $x=\left(\delta_{1}+\delta_{2}\right) / 2 \rho s, \varepsilon=T_{0}\left(v_{1}+v_{3}\right) /\left(\delta_{1}+\delta_{2}\right)$.

\section{Solution of the Problem}

By Helmholtz's theorem [27], the displacement vector $\vec{u}$ can be written in the form of the potentials $\phi\left(x_{1}, x_{3}, t\right)$ and $\psi\left(x_{1}, x_{3}, t\right)$ which are related to the displacement components $u_{1}$ and $u_{3}$ by the relations

$$
u_{1}=\frac{\partial \varphi}{\partial x_{1}}-\frac{\partial \psi}{\partial x_{3}}, \quad u_{3}=\frac{\partial \varphi}{\partial x_{3}}+\frac{\partial \psi}{\partial x_{1}}
$$

Substituting (3.1) into (2.12) and (2.13), we get the following wave equations satisfied by $\varphi, \psi, \xi, \eta$, and $\zeta$ :

$$
\left(c_{11}+P\right) \frac{\partial^{2} \varphi}{\partial x_{1}^{2}}+\left(c_{13}+2 c_{55}+P\right) \frac{\partial^{2} \varphi}{\partial x_{3}^{2}}-\rho g \frac{\partial \psi}{\partial x_{1}}-v_{1} T=\rho \frac{\partial^{2} \varphi}{\partial t^{2}},
$$




$$
\begin{gathered}
\frac{\partial}{\partial t}\left(\frac{\partial \zeta}{\partial x_{1}}-\frac{\partial \xi}{\partial x_{3}}\right)=0 \\
\left(c_{55}-\frac{P}{2}\right) \frac{\partial^{2} \psi}{\partial x_{1}^{2}}+\left(c_{33}-c_{31}-c_{55}-\frac{P}{2}\right) \frac{\partial^{2} \psi}{\partial x_{3}^{2}}+\rho g \frac{\partial \phi}{\partial x_{1}}+F \frac{\partial \eta}{\partial t}=\rho \frac{\partial^{2} \psi}{\partial t^{2}} \\
\nabla^{2} \xi-S \frac{\partial \xi}{\partial t}=0 \\
\nabla^{2} \eta-S \frac{\partial \eta}{\partial t}-\nabla^{4} \psi=0 \\
\nabla^{2} \zeta-S \frac{\partial \zeta}{\partial t}=0 \\
\nabla^{2} T-\frac{1}{x} \frac{\partial T}{\partial t}-\varepsilon \nabla^{2}\left(\frac{\partial \phi}{\partial t}\right)=0 \\
S=\frac{2 F}{M} .
\end{gathered}
$$

Eliminating $\eta$ from (3.4) and (3.6), we get

$$
\left(\nabla^{2}-S \frac{\partial}{\partial t}\right)\left[\left(c_{55}-\frac{P}{2}\right) \frac{\partial^{2} \psi}{\partial x_{1}^{2}}+\left(c_{33}-c_{31}-c_{55}-\frac{P}{2}\right) \frac{\partial^{2} \psi}{\partial x_{3}^{2}}-\rho \frac{\partial^{2} \psi}{\partial t^{2}}+\rho g \frac{\partial \phi}{\partial x_{1}}\right]+F \nabla^{4}\left(\frac{\partial \psi}{\partial t}\right)=0
$$

Also, $T$ can be eliminated by using (3.8) and (3.9) as follows:

$$
\left(\nabla^{2}-\frac{1}{x} \frac{\partial}{\partial t}\right)\left[\left(c_{11}+P\right) \frac{\partial^{2} \varphi}{\partial x_{1}^{2}}+\left(c_{13}+2 c_{55}+P\right) \frac{\partial^{2} \varphi}{\partial x_{3}^{2}}-\rho g \frac{\partial \psi}{\partial x_{1}}-\rho \frac{\partial^{2} \varphi}{\partial t^{2}}\right]-v_{1} \varepsilon \nabla^{2}\left(\frac{\partial \phi}{\partial t}\right)=0
$$

Assuming that

$$
(\varphi, \psi)=\left\{\varphi_{1}\left(x_{3}\right), \psi_{1}\left(x_{3}\right)\right\} \exp \left\{i\left(L x_{1}-b t\right)\right\},
$$


Mathematical Problems in Engineering

$$
(\xi, \eta, \zeta)=\left\{\xi_{1}\left(x_{3}\right), \eta_{1}\left(x_{3}\right), \zeta\left(x_{3}\right)\right\} \exp \left\{i\left(L x_{1}-b t\right)\right\},
$$

where $b$ is real positive and $L$ is in general complex.

Substituting (3.13) into (3.3), (3.5), and (3.7), we get

$$
\begin{gathered}
D \xi_{1}-i L \zeta_{1}=0, \\
D^{2} \xi_{1}+h^{2} \xi_{1}=0, \\
D^{2} \zeta_{1}+h^{2} \zeta_{1}=0,
\end{gathered}
$$

where $h^{2}=i b S-L^{2}, D \equiv d / d x$.

Solutions of (3.15) and (3.16) are

$$
\xi_{1}=A_{1} e^{i h x_{3}}+A_{2} e^{-i h x_{3}}, \quad \zeta_{1}=B_{1} e^{i h x_{3}}+B_{2} e^{-i h x_{3}},
$$

respectively.

From (3.14) and (3.17), we obtain

$$
h\left(A_{1} e^{i h x_{3}}-A_{2} e^{-i h x_{3}}\right)-L\left(B_{1} e^{i h x_{3}}-B_{2} e^{-i h x_{3}}\right)=0 .
$$

Equating the coefficients of $e^{i h x_{3}}$ and $e^{-i h x_{3}}$ to zero in (3.18), we get

$$
A_{1}=\frac{L}{h} B_{1}, \quad A_{2}=-\frac{L}{h} B_{2} .
$$

Substituting (3.12) and (3.13) into (3.10) and (3.11), we get

$$
\begin{aligned}
& {\left[\left(c_{33}-c_{13}-c_{55}-\frac{P}{2}-i b F\right) D^{4}\right.} \\
& +\left\{\left(i b S-L^{2}\right)\left(c_{33}-c_{13}-c_{55}-\frac{P}{2}\right)-L^{2}\left(c_{55}-\frac{P}{2}\right)+\rho b^{2}+2 i b F L^{2}\right\} D^{2} \\
& \left.+\left(i b S-L^{2}\right)\left(\rho b^{2}-L^{2}\left(c_{55}-\frac{P}{2}\right)-i b F L^{4}\right)\right] \psi_{1} \\
& \quad+i \rho g L\left[D^{2}-L^{2}+i b S\right] \phi_{1}=0,
\end{aligned}
$$




$$
\begin{aligned}
& \left\{\left(c_{13}+2 c_{55}+P\right) D^{4}\right. \\
& +\left[\rho b^{2}-L^{2}\left(c_{11}+c_{13}+2 c_{55}+2 P\right)+i b v_{1} \varepsilon+\frac{i b}{x}\left(c_{13}+2 c_{55}+P\right)\right] D^{2} \\
& \left.\quad+\left[\left(\frac{i b}{x}-L^{2}\right)\left(\rho b^{2}-L^{2}\left(c_{11}+P\right)\right)-i b v_{1} \varepsilon L^{2}\right]\right\} \phi_{1} \\
& \quad-i \rho g L\left[D^{2}-L^{2}+\frac{i b}{x}\right] \psi_{1}=0 .
\end{aligned}
$$

The solution of (3.20) and (3.21) has the form

$$
\begin{gathered}
\phi_{1}=A_{j} e^{-i \lambda_{j} x_{3}}+B_{j} e^{i \lambda_{j} x_{3}}, \\
\psi_{1}=E_{j} e^{-i \lambda_{j} x_{3}}+F_{j} e^{i \lambda_{j} x_{3}} \quad(j=3,4,5,6),
\end{gathered}
$$

where the constants $A_{j}, B_{j}$ are related with the constants $E_{j}, F_{j}$, respectively, by means of (3.20) or (3.21), and $\lambda_{j}(j=3,4,5,6)$ are taken to be imaginary.

Equating the coefficients of $e^{-i \lambda_{j} x_{3}}, e^{i \lambda_{j} x_{3}}(j=3,4,5,6)$ to zero, we have using (3.21)

$$
E_{j}=m_{j} A_{j}, \quad F_{j}=m_{j} B_{j} \quad(j=3,4,5,6),
$$

where

$$
\begin{aligned}
m_{j}= & \frac{1}{i \rho g L\left[-\lambda_{j}^{2}-L^{2}+i b / x\right]} \\
& \times\left\{\left(c_{13}+2 c_{55}+P\right) \lambda_{j}^{4}\right. \\
& \quad-\left[\rho b^{2}-L^{2}\left(c_{11}+c_{13}+2 c_{55}+2 P\right)+i b v_{1} \varepsilon+\frac{i b}{x}\left(c_{13}+2 c_{55}+P\right)\right] \lambda_{j}^{2} \\
& \left.+\left(\frac{i b}{x}-L^{2}\right)\left(\rho b^{2}-L^{2}\left(c_{11}+P\right)\right)-i b v_{1} \varepsilon L^{2}\right\},
\end{aligned}
$$

where $\lambda_{3}, \lambda_{4}, \lambda_{5}, \lambda_{6}$ are the imaginary roots of the equation

$$
k_{8} \lambda^{8}+k_{6} \lambda^{6}+k_{4} \lambda^{4}+k_{2} \lambda^{2}+k_{0}=0
$$




$$
\begin{aligned}
k_{8}= & \left(c_{13}+2 c_{55}+P\right)\left(c_{33}-c_{13}-c_{55}-\frac{P}{2}-i b F\right) \\
k_{6}= & -\left(\rho b^{2}-L^{2}\left(c_{11}+c_{13}+2 c_{55}+2 P\right)+i b v_{1} \varepsilon+\frac{i b}{x}\left(c_{13}+2 c_{55}+P\right)\right) \\
& \times\left(c_{33}-c_{13}-c_{55}-\frac{P}{2}-i b F\right)-\left(c_{13}+2 c_{55}+P\right) \\
& \times\left[\left(i b S-L^{2}\right)\left(c_{33}-c_{13}-c_{55}-\frac{P}{2}\right)-L^{2}\left(c_{55}-\frac{P}{2}\right)+\rho b^{2}+2 i b F L^{2}\right], \\
k_{4}= & \left(\frac{i b}{x}-L^{2}\right)\left(\rho b^{2}-L^{2}\left(c_{11}+P\right)-i b v_{1} \varepsilon L^{2}\right)\left(c_{33}-c_{13}-c_{55}-\frac{P}{2}-i b F\right) \\
& +\left(c_{13}+2 c_{55}+P\right)\left(i b S-L^{2}\right)\left[\rho b^{2}-L^{2}\left(c_{55}-\frac{P}{2}\right)-i b F L^{4}\right] \\
& +\left[\left(i b S-L^{2}\right)\left(c_{33}-c_{13}-c_{55}-\frac{P}{2}\right)+\rho b^{2}-L^{2}\left(c_{55}-\frac{P}{2}\right)+2 i b F L^{2}\right] \\
& \times\left[\left(i b S-L^{2}\right)\left[\rho b^{2}-L^{2}\left(c_{55}-\frac{P}{2}\right)-i b F L^{4}\right]-\rho^{2} g^{2} L^{2}\left(2 L^{2}-i b s-\frac{i b}{x}\right) .\right. \\
& \times\left[\rho b^{2}-L^{2}\left(c_{11}+c_{13}+2 c_{55}+2 P\right)+i b v_{1} \varepsilon+\frac{i b}{x}\left(c_{13}+2 c_{55}+P\right)\right]-\rho^{2} g^{2} L^{2}, \\
k_{0}= & \left(i b S-L^{2}\right)\left[\rho b^{2}-L^{2}\left(c_{55}-\frac{P}{2}\right)-i b F L^{4}\right] \\
& +\left[\left(i b S-L^{2}\right)\left(c_{33}-c_{13}-c_{55}-\frac{P}{2}\right)-L^{2}\left(c_{55}-\frac{P}{2}\right)+\rho b^{2}+2 i b F L^{2}\right] \\
k_{2}= & {\left[\left(\frac{i b}{x}-L^{2}\right)\left(\rho b^{2}-L^{2}\left(c_{11}+P\right)\right)-i b v_{1} \varepsilon L^{2}\right] } \\
& {\left.\left[c_{13}^{2}\left(c_{11}+P\right)\right)-i b v_{1} \varepsilon L^{2}\right]-\rho^{2} g^{2} L^{2}\left(\frac{i b}{x}-L^{2}\right)\left(i b S-L^{2}\right) . } \\
& \left.\left(c_{55}+2 P\right)+i b v_{1} \varepsilon+\frac{i b}{x}\left(c_{13}+2 c_{55}+P\right)\right]
\end{aligned}
$$

Using (3.2), (3.3), (3.12), (3.13), (3.22), and (3.23), we get

$$
\begin{gathered}
\phi=\left(A_{j} e^{-i \lambda_{j} x_{3}}+B_{j} e^{i \lambda_{j} x_{3}}\right) \exp \left(i\left(L x_{1}-b t\right)\right) \\
\psi=m_{j}\left(A_{j} e^{-i \lambda_{j} x_{3}}+B_{j} e^{i \lambda_{j} x_{3}}\right) \exp \left(i\left(L x_{1}-b t\right)\right) \quad(j=3,4,5,6)
\end{gathered}
$$


From (3.2) and (3.27), the temperature $T$ has the form

$$
T=n_{j}\left(A_{j} e^{-i \lambda_{j} x_{3}}+B_{j} e^{i \lambda_{j} x_{3}}\right) \exp \left(i\left(L x_{1}-b t\right)\right) \quad(j=3,4,5,6),
$$

where

$$
n_{j}=-\frac{1}{v_{1}}\left[\left(c_{13}+2 c_{55}+P\right) \lambda_{j}^{2}+L^{2}\left(c_{11}+P\right)+i L \rho g m_{j}-\rho b^{2}\right]
$$

also, from (3.6) and (3.27), $\eta$ has the form

$$
\eta=\Omega_{j} m_{j}\left(A_{j} e^{-i \lambda_{j} x_{3}}+B_{j} e^{i \lambda_{j} x_{3}}\right) \exp \left(i\left(L x_{1}-b t\right)\right) \quad(j=3,4,5,6),
$$

where

$$
\Omega_{j}=-\frac{\left(\lambda_{j}^{2}+L^{2}\right)^{2}}{\lambda_{j}^{2}+L^{2}-i b S} \quad(j=3,4,5,6) .
$$

We use the symbols with a bar for the quantities in the lower medium (except $\left.x_{3}, L, b, P, g\right)$, and by assuming that the solution of (3.20) and (3.21) satisfies the condition that the corresponding stresses vanish as $x_{3} \rightarrow-\infty$, we obtain

$$
\begin{gathered}
\overline{\xi_{1}}=-\frac{L}{\bar{h}} \overline{B_{2}} e^{-i \bar{h} x_{3}}, \quad \overline{\zeta_{1}}=\overline{B_{2}} e^{-i \bar{h} x_{3}}, \quad \overline{\eta_{1}}=\bar{\Omega} \overline{m_{j}} \overline{A_{j}} e^{-i \overline{\lambda_{j}} x_{3}}, \\
\overline{\phi_{1}}=\overline{A_{j}} e^{-i \overline{\lambda_{j}} x_{3}}, \quad \overline{\psi_{1}}=\overline{m_{j}} \overline{A_{j}} e^{-i \overline{\lambda_{j}} x_{3},} \\
\bar{T}=\overline{n_{j}} \overline{A_{j}} \exp \left(i\left(L x_{1}-b t-\overline{\lambda_{j}} x_{3}\right)\right) \quad(j=3,4,5,6) .
\end{gathered}
$$

\section{Boundary Conditions and Frequency Equation}

The boundary conditions on the interface $x_{3}=0$ are

$$
\begin{aligned}
& \begin{array}{lll}
\text { (i) } u_{1}=\overline{u_{1}}, & \text { (ii) } u_{3}=\overline{u_{3}}, \quad \text { (iii) } \xi=\bar{\xi},
\end{array} \\
& \text { (iv) } \eta=\bar{\eta}, \quad \text { (v) } \zeta=\bar{\zeta}, \quad \text { (vi) } M_{33}=\overline{M_{33}},
\end{aligned}
$$

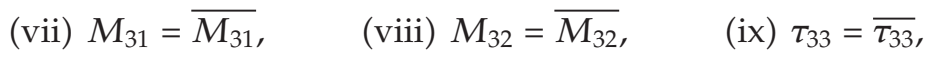

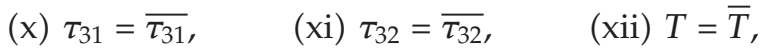

$$
\begin{aligned}
& \text { (xiii) } \frac{\partial T}{\partial x_{3}}+\theta T=\frac{\partial \bar{T}}{\partial x_{3}}+\bar{\theta} \bar{T} \text {. }
\end{aligned}
$$


The boundary conditions on the free surface $x_{3}=H$ are

$$
\begin{array}{rll}
\text { (xiv) } M_{33}=0, & (\text { xv }) M_{31}=0, & (\text { xvi }) M_{32}=0, \\
(\text { xvii }) \tau_{33}=0, & (\text { xviii }) \tau_{31}=0, & (\text { xix }) \tau_{32}=0, \\
(x x) \frac{\partial T}{\partial x_{3}}+\theta T=0, &
\end{array}
$$

where

$$
\begin{gathered}
M_{33}=M \frac{\partial \zeta}{\partial x_{3}}, \quad M_{32}=M \frac{\partial}{\partial x_{3}}\left(\eta-\nabla^{2} \psi\right), \quad M_{31}=M \frac{\partial \xi}{\partial x_{3}}, \\
\tau_{33}=c_{13} \frac{\partial^{2} \varphi}{\partial x_{1}^{2}}+c_{33} \frac{\partial^{2} \varphi}{\partial x_{3}^{2}}+\left(c_{33}-c_{13}\right) \frac{\partial^{2} \psi}{\partial x_{1} \partial x_{3}}-v_{3} T, \quad \tau_{32}=-F \frac{\partial \xi}{\partial t} \\
\tau_{31}=c_{55}\left(\frac{\partial^{2} \psi}{\partial x_{1}^{2}}-\frac{\partial^{2} \psi}{\partial x_{3}^{2}}+2 \frac{\partial^{2} \varphi}{\partial x_{1} \partial x_{3}}\right)-F \frac{\partial \eta}{\partial t}
\end{gathered}
$$

$\theta$ is the ratio of the coefficients of heat transfer to the thermal conductivity.

From the boundary conditions (iii), (v), (vi), and (vii), we get

$$
\begin{gathered}
B_{1} e^{i h H}-B_{2} e^{-i h H}=-\overline{B_{2}} e^{-i \bar{h} H}, \\
B_{1} e^{i h H}+B_{2} e^{-i h H}=-\overline{B_{2}} e^{i \bar{h} H}, \\
M\left(B_{1} e^{i h H}-B_{2} e^{-i h H}\right)=-\overline{M B_{2}} e^{-i \bar{h} H}, \\
M\left(B_{1} e^{i h H}+B_{2} e^{-i h H}\right)=-\overline{M B_{2}} e^{i \bar{h} H},
\end{gathered}
$$

hence

$$
B_{1}=B_{2}=\overline{B_{2}}=0, \quad \xi=\zeta=\bar{\xi}=\bar{\zeta}=0
$$

The other significant boundary conditions are responsible for the following relations:

$$
\begin{gathered}
\text { (xvi) } \quad\left(\lambda_{j}^{2}+\Omega_{j}+L^{2}\right)\left(e^{-i \lambda_{j} H} A_{j}-e^{i \lambda_{j} H} B_{j}\right)=0, \\
(\text { xvii }) \quad\left(c_{13} L\left(L+m_{j} \lambda_{j}\right)+c_{33} \lambda_{j}\left(\lambda_{j}-L m_{j}\right)+v_{3} n_{j}\right) e^{-i \lambda_{j} H} A_{j} \\
\quad+\left(c_{13} L\left(L-m_{j} \lambda_{j}\right)+c_{33} \lambda_{j}\left(\lambda_{j}+L m_{j}\right)+v_{3} n_{j}\right) e^{i \lambda_{j} H} B_{j}=0,
\end{gathered}
$$


(xviii)

$$
\begin{aligned}
& {\left[c_{55}\left(\lambda_{j}^{2}-L^{2}\right) m_{j}+2 L \lambda_{j}+i b F \Omega_{j} m_{j}\right] e^{-i \lambda_{j} H} A_{j}} \\
& \quad+\left[c_{55}\left(\lambda_{j}^{2}-L^{2}\right) m_{j}-2 L \lambda_{j}+i b F \Omega_{j} m_{j}\right] e^{i \lambda_{j} H} B_{j}=0,
\end{aligned}
$$

(i) $\left(L+m_{j} \lambda_{j}\right) A_{j}+\left(L-m_{j} \lambda_{j}\right) B_{j}=\left(L+\overline{m_{j}} \overline{\lambda_{j}}\right) \overline{A_{j}}$,

(ii) $\quad\left(L m_{j}-\lambda_{j}\right) A_{j}+\left(L m_{j}+\lambda_{j}\right) B_{j}=\left(L \overline{m_{j}}-\overline{\lambda_{j}}\right) \overline{A_{j}}$,

(iv) $\Omega_{j} m_{j}\left(A_{j}+B_{j}\right)=\overline{\Omega_{j}} \overline{m_{j}} \overline{A_{j}}$,

(viii) $M m_{j} \lambda_{j}\left(\lambda_{j}^{2}+\Omega_{j}+L^{2}\right)\left(A_{j}-B_{j}\right)=\bar{M} \overline{m_{j}} \overline{\lambda_{j}}\left(\overline{\lambda_{j}^{2}}+\overline{\Omega_{j}}+L^{2}\right)$,

(ix) $\quad\left[c_{13} L\left(L+m_{j} \lambda_{j}\right)+c_{33} \lambda_{j}\left(\lambda_{j}-L m_{j}\right)+v_{3} n_{j}\right] A_{j}$

$+\left[c_{13} L\left(L-m_{j} \lambda_{j}\right)+c_{33} \lambda_{j}\left(\lambda_{j}+L m_{j}\right)+v_{3} n_{j}\right] B_{j}$

$=\left[\overline{c_{13}} L\left(L+\overline{m_{j}} \overline{\lambda_{j}}\right)+\overline{c_{33}} \overline{\lambda_{j}}\left(\overline{\lambda_{j}}-L \overline{m_{j}}\right)+\overline{v_{3} n_{j}}\right] \overline{A_{j}}$,

(x) $\quad\left\{c_{55}\left[m_{j}\left(L^{2}+\lambda_{j}^{2}\right)-2 L \lambda_{j}\right]-i b F m_{j} \Omega_{j}\right\} A_{j}$

$$
\begin{aligned}
& +\left\{c_{55}\left[m_{j}\left(L^{2}+\lambda_{j}^{2}\right)+2 L \lambda_{j}\right]-i b F m_{j} \Omega_{j}\right\} B_{j} \\
& =\left\{\overline{C_{55}}\left[\overline{m_{j}}\left(L^{2}+\overline{\lambda_{j}^{2}}\right)-2 L \overline{\lambda_{j}}\right]-i b \bar{F} \overline{m_{j}} \overline{\Omega_{j}}\right\} \overline{A_{j}},
\end{aligned}
$$

(xii) $\quad n_{j} A_{j}+n_{j} B_{j}=\overline{n_{j}} \overline{A_{j}}$,

(xiii) $\quad n_{j}\left(\theta-i \lambda_{j}\right) A_{j}+n_{j}\left(\theta+i \lambda_{j}\right) B_{j}=\overline{n_{j}}\left(\bar{\theta}-i \overline{\lambda_{j}}\right) \overline{A_{j}}$,

(xx) $\quad n_{j}\left(\theta-i \lambda_{j}\right) e^{-i \lambda_{j} H} A_{j}+n_{j}\left(\theta+i \lambda_{j}\right) e^{i \lambda_{j} H} B_{j}=0, \quad(j=3,4,5,6)$.

Elimination of $A_{j}, B_{j}, \overline{A_{j}}(j=3,4,5,6)$ gives the wave velocity equation in the form

$$
\operatorname{det} \cdot\left(d_{r c}\right)=0 \quad(r, c=1,2, \ldots, 12)
$$

where the nonvanishing entries of the twelfth-order determinant of $d_{r c}$ are given by

$$
\begin{array}{ll}
d_{11}=\left(\lambda_{3}^{2}+\Omega_{3}+L^{2}\right) e^{-i \lambda_{3} H}, & d_{12}=\left(\lambda_{4}^{2}+\Omega_{4}+L^{2}\right) e^{-i \lambda_{4} H}, \\
d_{13}=\left(\lambda_{5}^{2}+\Omega_{5}+L^{2}\right) e^{-i \lambda_{5} H}, & d_{14}=\left(\lambda_{6}^{2}+\Omega_{6}+L^{2}\right) e^{-i \lambda_{6} H},
\end{array}
$$


Mathematical Problems in Engineering

$$
\begin{aligned}
& d_{15}=-\left(\lambda_{3}^{2}+\Omega_{3}+L^{2}\right) e^{i \lambda_{3} H}, \quad d_{16}=-\left(\lambda_{4}^{2}+\Omega_{4}+L^{2}\right) e^{i \lambda_{4} H}, \\
& d_{17}=-\left(\lambda_{5}^{2}+\Omega_{5}+L^{2}\right) e^{i \lambda_{5} H}, \quad d_{18}=-\left(\lambda_{6}^{2}+\Omega_{6}+L^{2}\right) e^{i \lambda_{6} H}, \\
& d_{21}=\left(c_{13} L\left(L+m_{3} \lambda_{3}\right)+c_{33} \lambda_{3}\left(\lambda_{3}-L m_{3}\right)+v_{3} n_{3}\right) e^{-i \lambda_{3} H}, \\
& d_{22}=\left(c_{13} L\left(L+m_{4} \lambda_{4}\right)+c_{33} \lambda_{4}\left(\lambda_{4}-L m_{4}\right)+v_{3} n_{4}\right) e^{-i \lambda_{4} H}, \\
& d_{23}=\left(c_{13} L\left(L+m_{5} \lambda_{5}\right)+c_{33} \lambda_{5}\left(\lambda_{5}-L m_{5}\right)+v_{3} n_{5}\right) e^{-i \lambda_{5} H}, \\
& d_{24}=\left(c_{13} L\left(L+m_{6} \lambda_{6}\right)+c_{33} \lambda_{6}\left(\lambda_{6}-L m_{6}\right)+v_{3} n_{6}\right) e^{-i \lambda_{6} H}, \\
& d_{25}=\left(c_{13} L\left(L-m_{3} \lambda_{3}\right)+c_{33} \lambda_{3}\left(\lambda_{3}+L m_{3}\right)+v_{3} n_{3}\right) e^{i \lambda_{3} H}, \\
& d_{26}=\left(c_{13} L\left(L-m_{4} \lambda_{4}\right)+c_{33} \lambda_{4}\left(\lambda_{4}+L m_{4}\right)+v_{3} n_{4}\right) e^{i \lambda_{4} H}, \\
& d_{27}=\left(c_{13} L\left(L-m_{5} \lambda_{5}\right)+c_{33} \lambda_{5}\left(\lambda_{5}+L m_{5}\right)+v_{3} n_{5}\right) e^{i \lambda_{5} H}, \\
& d_{28}=\left(c_{13} L\left(L-m_{6} \lambda_{6}\right)+c_{33} \lambda_{6}\left(\lambda_{6}+L m_{6}\right)+v_{3} n_{6}\right) e^{i \lambda_{6} H}, \\
& d_{31}=\left[c_{55}\left(\lambda_{3}^{2}-L^{2}\right) m_{3}+2 L \lambda_{3}+i b F \Omega_{3} m_{3}\right] e^{-i \lambda_{3} H}, \\
& d_{32}=\left[c_{55}\left(\lambda_{4}^{2}-L^{2}\right) m_{4}+2 L \lambda_{4}+i b F \Omega_{4} m_{4}\right] e^{-i \lambda_{4} H}, \\
& d_{33}=\left[c_{55}\left(\lambda_{5}^{2}-L^{2}\right) m_{5}+2 L \lambda_{5}+i b F \Omega_{5} m_{5}\right] e^{-i \lambda_{5} H}, \\
& d_{34}=\left[c_{55}\left(\lambda_{6}^{2}-L^{2}\right) m_{6}+2 L \lambda_{6}+i b F \Omega_{6} m_{6}\right] e^{-i \lambda_{6} H}, \\
& d_{35}=\left[c_{55}\left(\lambda_{3}^{2}-L^{2}\right) m_{3}-2 L \lambda_{3}+i b F \Omega_{3} m_{3}\right] e^{i \lambda_{3} H}, \\
& d_{36}=\left[c_{55}\left(\lambda_{4}^{2}-L^{2}\right) m_{4}-2 L \lambda_{4}+i b F \Omega_{4} m_{4}\right] e^{i \lambda_{4} H}, \\
& d_{37}=\left[c_{55}\left(\lambda_{5}^{2}-L^{2}\right) m_{5}-2 L \lambda_{5}+i b F \Omega_{5} m_{5}\right] e^{i \lambda_{5} H}, \\
& d_{38}=\left[c_{55}\left(\lambda_{6}^{2}-L^{2}\right) m_{6}-2 L \lambda_{6}+i b F \Omega_{6} m_{6}\right] e^{i \lambda_{6} H}, \\
& d_{41}=L+m_{3} \lambda_{3}, \quad d_{42}=L+m_{4} \lambda_{4}, \\
& d_{43}=L+m_{5} \lambda_{5}, \quad d_{44}=L+m_{6} \lambda_{6}, \\
& d_{45}=L-m_{3} \lambda_{3}, \quad d_{46}=L-m_{4} \lambda_{4}, \\
& d_{47}=L-m_{5} \lambda_{5}, \quad d_{48}=L-m_{6} \lambda_{6}, \\
& d_{49}=-\left(L+\overline{m_{3}} \overline{\lambda_{3}}\right), \quad d_{410}=-\left(L+\overline{m_{4}} \overline{\lambda_{4}}\right), \\
& d_{411}=-\left(L+\overline{m_{5}} \overline{\lambda_{5}}\right), \quad d_{412}=-\left(L+\overline{m_{6}} \overline{\lambda_{6}}\right), \\
& d_{51}=L m_{3}-\lambda_{3}, \quad d_{52}=L m_{4}-\lambda_{4}, \\
& d_{53}=L m_{5}-\lambda_{5}, \quad d_{54}=L m_{6}-\lambda_{6},
\end{aligned}
$$




$$
\begin{aligned}
& d_{55}=L m_{3}+\lambda_{3}, \quad d_{56}=L m_{4}+\lambda_{4}, \\
& d_{57}=L m_{5}+\lambda_{5}, \quad d_{58}=L m_{6}+\lambda_{6}, \\
& d_{59}=-L \overline{m_{3}}+\overline{\lambda_{3}}, \quad d_{510}=-L \overline{m_{4}}+\overline{\lambda_{4}}, \\
& d_{511}=-L \overline{m_{5}}+\overline{\lambda_{5}}, \quad d_{512}=-L \overline{m_{6}}+\overline{\lambda_{6}}, \\
& d_{61}=\Omega_{3} m_{3}, \quad d_{62}=\Omega_{4} m_{4}, \quad d_{63}=\Omega_{5} m_{5}, \quad d_{64}=\Omega_{6} m_{6}, \\
& d_{65}=\Omega_{3} m_{3}, \quad d_{66}=\Omega_{4} m_{4}, \quad d_{67}=\Omega_{5} m_{5}, \quad d_{68}=\Omega_{6} m_{6}, \\
& d_{69}=-\overline{\Omega_{3}} \overline{m_{3}}, \quad d_{610}=-\overline{\Omega_{4}} \overline{m_{4}}, \quad d_{611}=-\overline{\Omega_{5}} \overline{m_{5}}, \quad d_{612}=-\overline{\Omega_{6}} \overline{m_{6}}, \\
& d_{71}=M m_{3} \lambda_{3}\left(\lambda_{3}^{2}+\Omega_{3}+L^{2}\right), \quad d_{72}=M m_{4} \lambda_{4}\left(\lambda_{4}^{2}+\Omega_{4}+L^{2}\right), \\
& d_{73}=M m_{5} \lambda_{5}\left(\lambda_{5}^{2}+\Omega_{5}+L^{2}\right), \quad d_{74}=M m_{6} \lambda_{6}\left(\lambda_{6}^{2}+\Omega_{6}+L^{2}\right), \\
& d_{75}=-M m_{3} \lambda_{3}\left(\lambda_{3}^{2}+\Omega_{3}+L^{2}\right), \quad d_{76}=-M m_{4} \lambda_{4}\left(\lambda_{4}^{2}+\Omega_{4}+L^{2}\right), \\
& d_{77}=-M m_{5} \lambda_{5}\left(\lambda_{5}^{2}+\Omega_{5}+L^{2}\right), \quad d_{78}=-M m_{6} \lambda_{6}\left(\lambda_{6}^{2}+\Omega_{6}+L^{2}\right) ， \\
& d_{79}=-\bar{M} \overline{m_{3}} \overline{\lambda_{3}}\left(\overline{\lambda_{3}^{2}}+\overline{\Omega_{3}}+L^{2}\right), \quad d_{710}=-\bar{M} \overline{m_{4}} \overline{\lambda_{4}}\left(\overline{\lambda_{4}^{2}}+\overline{\Omega_{4}}+L^{2}\right), \\
& d_{711}=-\bar{M} \overline{m_{5}} \overline{\lambda_{5}}\left(\overline{\lambda_{5}^{2}}+\overline{\Omega_{5}}+L^{2}\right), \quad d_{712}=-\bar{M} \overline{m_{6}} \overline{\lambda_{6}}\left(\overline{\lambda_{6}^{2}}+\overline{\Omega_{6}}+L^{2}\right), \\
& d_{81}=c_{13} L\left(L+m_{3} \lambda_{3}\right)+c_{33} \lambda_{3}\left(\lambda_{3}-L m_{3}\right)+v_{3} n_{3}, \\
& d_{82}=c_{13} L\left(L+m_{4} \lambda_{4}\right)+c_{33} \lambda_{4}\left(\lambda_{4}-L m_{4}\right)+v_{3} n_{4}, \\
& d_{83}=c_{13} L\left(L+m_{5} \lambda_{5}\right)+c_{33} \lambda_{5}\left(\lambda_{5}-L m_{5}\right)+v_{3} n_{5}, \\
& d_{84}=c_{13} L\left(L+m_{6} \lambda_{6}\right)+c_{33} \lambda_{6}\left(\lambda_{6}-L m_{6}\right)+v_{3} n_{6}, \\
& d_{85}=c_{13} L\left(L-m_{3} \lambda_{3}\right)+c_{33} \lambda_{3}\left(\lambda_{3}+L m_{3}\right)+v_{3} n_{3}, \\
& d_{86}=c_{13} L\left(L-m_{4} \lambda_{4}\right)+c_{33} \lambda_{4}\left(\lambda_{4}+L m_{4}\right)+v_{3} n_{4} \\
& d_{87}=c_{13} L\left(L-m_{5} \lambda_{5}\right)+c_{33} \lambda_{5}\left(\lambda_{5}+L m_{5}\right)+v_{3} n_{5}, \\
& d_{88}=c_{13} L\left(L-m_{6} \lambda_{6}\right)+c_{33} \lambda_{6}\left(\lambda_{6}+L m_{6}\right)+v_{3} n_{6}, \\
& d_{89}=-\left[\overline{c_{13}} L\left(L+\overline{m_{3}} \overline{\lambda_{3}}\right)+\overline{c_{33}} \overline{\lambda_{3}}\left(\overline{\lambda_{3}}-L \overline{m_{3}}\right)+\overline{v_{3} n_{3}}\right], \\
& d_{810}=-\left[\overline{c_{13}} L\left(L+\overline{m_{4}} \overline{\lambda_{4}}\right)+\overline{c_{33}} \overline{\lambda_{4}}\left(\overline{\lambda_{4}}-L \overline{m_{4}}\right)+\overline{v_{3} n_{4}}\right], \\
& d_{811}=-\left[\overline{c_{13}} L\left(L+\overline{m_{5}} \overline{\lambda_{5}}\right)+\overline{c_{33}} \overline{\lambda_{5}}\left(\overline{\lambda_{5}}-L \overline{m_{5}}\right)+\overline{v_{3} n_{5}}\right], \\
& d_{812}=-\left[\overline{c_{13}} L\left(L+\overline{m_{6}} \overline{\lambda_{6}}\right)+\overline{c_{33}} \overline{\lambda_{6}}\left(\overline{\lambda_{6}}-L \overline{m_{6}}\right)+\overline{v_{3} n_{6}}\right] \text {, } \\
& d_{91}=c_{55}\left[m_{3}\left(L^{2}+\lambda_{3}^{2}\right)-2 L \lambda_{3}\right]-i b F m_{3} \Omega_{3},
\end{aligned}
$$


Mathematical Problems in Engineering

$$
\begin{aligned}
& d_{92}=c_{55}\left[m_{4}\left(L^{2}+\lambda_{4}^{2}\right)-2 L \lambda_{4}\right]-i b F m_{4} \Omega_{4}, \\
& d_{93}=c_{55}\left[m_{5}\left(L^{2}+\lambda_{5}^{2}\right)-2 L \lambda_{5}\right]-i b F m_{5} \Omega_{5}, \\
& d_{94}=c_{55}\left[m_{6}\left(L^{2}+\lambda_{6}^{2}\right)-2 L \lambda_{6}\right]-i b F m_{6} \Omega_{6}, \\
& d_{95}=c_{55}\left[m_{3}\left(L^{2}+\lambda_{3}^{2}\right)+2 L \lambda_{3}\right]-i b F m_{3} \Omega_{3}, \\
& d_{96}=c_{55}\left[m_{4}\left(L^{2}+\lambda_{4}^{2}\right)+2 L \lambda_{4}\right]-i b F m_{4} \Omega_{4}, \\
& d_{97}=c_{55}\left[m_{5}\left(L^{2}+\lambda_{5}^{2}\right)+2 L \lambda_{5}\right]-i b F m_{5} \Omega_{5}, \\
& d_{98}=c_{55}\left[m_{6}\left(L^{2}+\lambda_{6}^{2}\right)+2 L \lambda_{6}\right]-i b F m_{6} \Omega_{6}, \\
& d_{99}=-\left\{\overline{\bar{C}_{55}}\left[\overline{m_{3}}\left(L^{2}+\overline{\lambda_{3}^{2}}\right)-2 L \overline{\lambda_{3}}\right]-i b \bar{F} \overline{m_{3}} \overline{\Omega_{3}}\right\}, \\
& d_{910}=-\left\{\overline{C_{55}}\left[\overline{m_{4}}\left(L^{2}+\overline{\lambda_{4}^{2}}\right)-2 L \overline{\lambda_{4}}\right]-i b \bar{F} \overline{m_{4}} \overline{\Omega_{4}}\right\}, \\
& d_{911}=-\left\{\overline{C_{55}}\left[\overline{m_{5}}\left(L^{2}+\overline{\lambda_{5}^{2}}\right)-2 L \overline{\lambda_{5}}\right]-i b \bar{F} \overline{m_{5}} \overline{\Omega_{5}}\right\} \text {, } \\
& d_{912}=-\left\{\overline{c_{55}}\left[\overline{m_{6}}\left(L^{2}+\overline{\lambda_{6}^{2}}\right)-2 L \overline{\lambda_{6}}\right]-i b \bar{F} \overline{m_{6}} \overline{\Omega_{6}}\right\}, \\
& d_{101}=n_{3}, \quad d_{102}=n_{4}, \quad d_{103}=n_{5}, \quad d_{104}=n_{6}, \\
& d_{105}=n_{3}, \quad d_{106}=n_{4}, \quad d_{107}=n_{5}, \quad d_{108}=n_{6}, \\
& d_{109}=\overline{n_{3}}, \quad d_{1010}=\overline{n_{4}}, \quad d_{1011}=\overline{n_{5}}, \quad d_{1012}=\overline{n_{6}}, \\
& d_{111}=n_{3}\left(\theta-i \lambda_{3}\right), \quad d_{112}=n_{4}\left(\theta-i \lambda_{4}\right), \quad d_{113}=n_{5}\left(\theta-i \lambda_{5}\right), \\
& d_{114}=n_{6}\left(\theta-i \lambda_{6}\right), \quad d_{115}=n_{3}\left(\theta+i \lambda_{3}\right), \quad d_{116}=n_{4}\left(\theta+i \lambda_{4}\right), \\
& d_{117}=n_{5}\left(\theta+i \lambda_{5}\right), \quad d_{118}=n_{6}\left(\theta+i \lambda_{6}\right), \quad d_{119}=-\overline{n_{3}}\left(\bar{\theta}-i \overline{\lambda_{3}}\right), \\
& d_{1110}=-\overline{n_{4}}\left(\bar{\theta}-i \overline{\lambda_{4}}\right), \quad d_{1111}=-\overline{n_{5}}\left(\bar{\theta}-i \overline{\lambda_{5}}\right), \quad d_{1112}=-\overline{n_{6}}\left(\bar{\theta}-i \overline{\lambda_{6}}\right), \\
& d_{121}=n_{3}\left(\theta-i \lambda_{3}\right) e^{-i \lambda_{3} H}, \quad d_{122}=n_{4}\left(\theta-i \lambda_{4}\right) e^{-i \lambda_{4} H}, \\
& d_{123}=n_{5}\left(\theta-i \lambda_{5}\right) e^{-i \lambda_{5} H}, \quad d_{124}=n_{6}\left(\theta-i \lambda_{6}\right) e^{-i \lambda_{6} H}, \\
& d_{125}=n_{3}\left(\theta+i \lambda_{3}\right) e^{i \lambda_{3} H}, \quad d_{126}=n_{4}\left(\theta+i \lambda_{4}\right) e^{i \lambda_{4} H}, \\
& d_{127}=n_{5}\left(\theta+i \lambda_{5}\right) e^{i \lambda_{5} H}, \quad d_{128}=n_{6}\left(\theta+i \lambda_{6}\right) e^{i \lambda_{6} H} .
\end{aligned}
$$

Equation (4.7) determines the wave velocity equation for the Rayleigh waves in an orthotropic thermoelastic granular medium under the influence of initial stress and gravity field. 


\section{Stoneley Waves}

To investigate the possibility Stoneley waves in thermoelastic granular medium under the influence of initial stress and gravity field, we replace the layer by a half-space $(H \rightarrow \infty)$, in the preceding problem, in this case $e^{i \lambda_{j} H} \rightarrow 0$, and the coefficients of $e^{-i \lambda_{j} H}(j=3,4,5,6)$ must vanish. Hence, the wave velocity equation (4.7) reduces to

$$
\operatorname{det} \cdot\left(d_{r c}^{\prime}\right)=0 \quad(r, c=1,2, \ldots, 8),
$$

where

$$
\begin{aligned}
& d_{11}^{\prime}=L+m_{3} \lambda_{3}, \quad d_{12}^{\prime}=L+m_{4} \lambda_{4}, \\
& d_{13}^{\prime}=L+m_{5} \lambda_{5}, \quad d_{14}^{\prime}=L+m_{6} \lambda_{6}, \\
& d_{15}^{\prime}=L-m_{3} \lambda_{3}, \quad d_{16}^{\prime}=L-m_{4} \lambda_{4}, \\
& d_{17}^{\prime}=L-m_{5} \lambda_{5}, \quad d_{18}^{\prime}=L-m_{6} \lambda_{6}, \\
& d_{21}^{\prime}=L m_{3}-\lambda_{3}, \quad d_{22}^{\prime}=L m_{4}-\lambda_{4}, \\
& d_{23}^{\prime}=L m_{5}-\lambda_{5}, \quad d_{24}^{\prime}=L m_{6}-\lambda_{6}, \\
& d_{25}^{\prime}=L m_{3}+\lambda_{3}, \quad d_{26}^{\prime}=L m_{4}+\lambda_{4}, \\
& d_{27}^{\prime}=L m_{5}+\lambda_{5}, \quad d_{28}^{\prime}=L m_{6}+\lambda_{6}, \\
& d_{31}^{\prime}=\Omega_{3} m_{3}, \quad d_{32}^{\prime}=\Omega_{4} m_{4}, \quad d_{33}^{\prime}=\Omega_{5} m_{5}, \quad d_{34}^{\prime}=\Omega_{6} m_{6}, \\
& d_{35}^{\prime}=-\Omega_{3} m_{3}, \quad d_{36}^{\prime}=-\Omega_{4} m_{4}, \quad d_{37}^{\prime}=-\Omega_{5} m_{5}, \quad d_{38}^{\prime}=-\Omega_{6} m_{6}, \\
& d_{41}^{\prime}=M m_{3} \lambda_{3}\left(\lambda_{3}^{2}+\Omega_{3}+L^{2}\right), \quad d_{42}^{\prime}=M m_{4} \lambda_{4}\left(\lambda_{4}^{2}+\Omega_{4}+L^{2}\right), \\
& d_{43}^{\prime}=M m_{5} \lambda_{5}\left(\lambda_{5}^{2}+\Omega_{5}+L^{2}\right), \quad d_{44}^{\prime}=M m_{6} \lambda_{6}\left(\lambda_{6}^{2}+\Omega_{6}+L^{2}\right), \\
& d_{45}^{\prime}=-M m_{3} \lambda_{3}\left(\lambda_{3}^{2}+\Omega_{3}+L^{2}\right), \quad d_{46}^{\prime}=-M m_{4} \lambda_{4}\left(\lambda_{4}^{2}+\Omega_{4}+L^{2}\right), \\
& d_{47}^{\prime}=-M m_{5} \lambda_{5}\left(\lambda_{5}^{2}+\Omega_{5}+L^{2}\right), \quad d_{48}^{\prime}=-M m_{6} \lambda_{6}\left(\lambda_{6}^{2}+\Omega_{6}+L^{2}\right), \\
& d_{51}^{\prime}=c_{13} L\left(L+m_{3} \lambda_{3}\right)+c_{33} \lambda_{3}\left(\lambda_{3}-L m_{3}\right)+v_{3} n_{3}, \\
& d_{52}^{\prime}=c_{13} L\left(L+m_{4} \lambda_{4}\right)+c_{33} \lambda_{4}\left(\lambda_{4}-L m_{4}\right)+v_{3} n_{4}, \\
& d_{53}^{\prime}=c_{13} L\left(L+m_{5} \lambda_{5}\right)+c_{33} \lambda_{5}\left(\lambda_{5}-L m_{5}\right)+v_{3} n_{5}, \\
& d_{54}^{\prime}=c_{13} L\left(L+m_{6} \lambda_{6}\right)+c_{33} \lambda_{6}\left(\lambda_{6}-L m_{6}\right)+v_{3} n_{6}, \\
& d_{55}^{\prime}=c_{13} L\left(L-m_{3} \lambda_{3}\right)+c_{33} \lambda_{3}\left(\lambda_{3}+L m_{3}\right)+v_{3} n_{3},
\end{aligned}
$$




$$
\begin{aligned}
& d_{56}^{\prime}=c_{13} L\left(L-m_{4} \lambda_{4}\right)+c_{33} \lambda_{4}\left(\lambda_{4}+L m_{4}\right)+v_{3} n_{4}, \\
& d_{57}^{\prime}=c_{13} L\left(L-m_{5} \lambda_{5}\right)+c_{33} \lambda_{5}\left(\lambda_{5}+L m_{5}\right)+v_{3} n_{5}, \\
& d_{58}^{\prime}=c_{13} L\left(L-m_{6} \lambda_{6}\right)+c_{33} \lambda_{6}\left(\lambda_{6}+L m_{6}\right)+v_{3} n_{6}, \\
& d_{61}^{\prime}=c_{55}\left[m_{3}\left(L^{2}+\lambda_{3}^{2}\right)-2 L \lambda_{3}\right]-i b F m_{3} \Omega_{3}, \\
& d_{62}^{\prime}=c_{55}\left[m_{4}\left(L^{2}+\lambda_{4}^{2}\right)-2 L \lambda_{4}\right]-i b F m_{4} \Omega_{4}, \\
& d_{63}^{\prime}=c_{55}\left[m_{5}\left(L^{2}+\lambda_{5}^{2}\right)-2 L \lambda_{5}\right]-i b F m_{5} \Omega_{5}, \\
& d_{64}^{\prime}=c_{55}\left[m_{6}\left(L^{2}+\lambda_{6}^{2}\right)-2 L \lambda_{6}\right]-i b F m_{6} \Omega_{6}, \\
& d_{65}^{\prime}=c_{55}\left[m_{3}\left(L^{2}+\lambda_{3}^{2}\right)+2 L \lambda_{3}\right]-i b F m_{3} \Omega_{3}, \\
& d_{66}^{\prime}=c_{55}\left[m_{4}\left(L^{2}+\lambda_{4}^{2}\right)+2 L \lambda_{4}\right]-i b F m_{4} \Omega_{4}, \\
& d_{67}^{\prime}=c_{55}\left[m_{5}\left(L^{2}+\lambda_{5}^{2}\right)+2 L \lambda_{5}\right]-i b F m_{5} \Omega_{5}, \\
& d_{68}^{\prime}=c_{55}\left[m_{6}\left(L^{2}+\lambda_{6}^{2}\right)+2 L \lambda_{6}\right]-i b F m_{6} \Omega_{6}, \\
& d_{71}^{\prime}=n_{3}, \quad d_{72}^{\prime}=n_{4}, \quad d_{73}^{\prime}=n_{5}, \quad d_{74}^{\prime}=n_{6}, \\
& d_{75}^{\prime}=n_{3}, \quad d_{76}^{\prime}=n_{4}, \quad d_{77}^{\prime}=n_{5}, \quad d_{78}^{\prime}=n_{6}, \\
& d_{81}^{\prime}=n_{3}\left(\theta-i \lambda_{3}\right), \quad d_{82}^{\prime}=n_{4}\left(\theta-i \lambda_{4}\right), \quad d_{83}^{\prime}=n_{5}\left(\theta-i \lambda_{5}\right), \\
& d_{84}^{\prime}=n_{6}\left(\theta-i \lambda_{6}\right), \quad d_{85}^{\prime}=n_{3}\left(\theta+i \lambda_{3}\right), \quad d_{86}^{\prime}=n_{4}\left(\theta+i \lambda_{4}\right), \\
& d_{87}^{\prime}=n_{5}\left(\theta+i \lambda_{5}\right), \quad d_{88}^{\prime}=n_{6}\left(\theta+i \lambda_{6}\right) .
\end{aligned}
$$

Equation (5.1) determines the wave velocity equation for the Stoneley waves in an orthotropic thermoelastic granular medium under the influence of initial stress and gravity field.

\section{Special Cases}

The transcendental equations (4.7) and (5.1), in the determinant form, have complex roots. The real part gives the velocity of Rayleigh waves and Stoneley waves, respectively, while the imaginary part gives the attenuation due to the granular nature of the medium. It is clear from the frequency equations (4.7) and (5.1) that the phase velocity depends on the initial stress $P$, the friction $F$, the gravity field, and the coupling factor $\varepsilon$. 


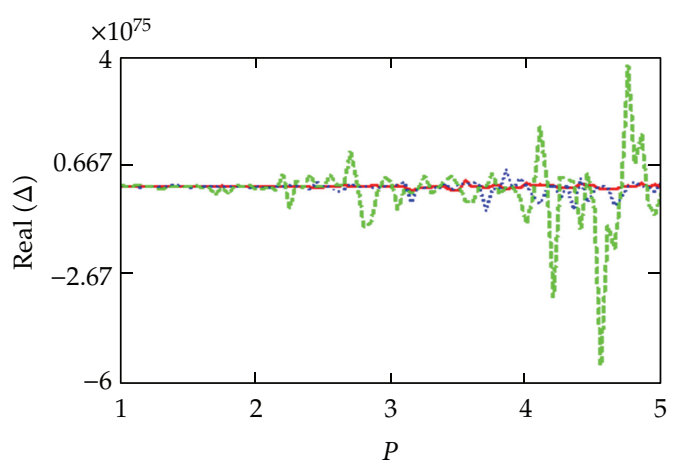

(a)

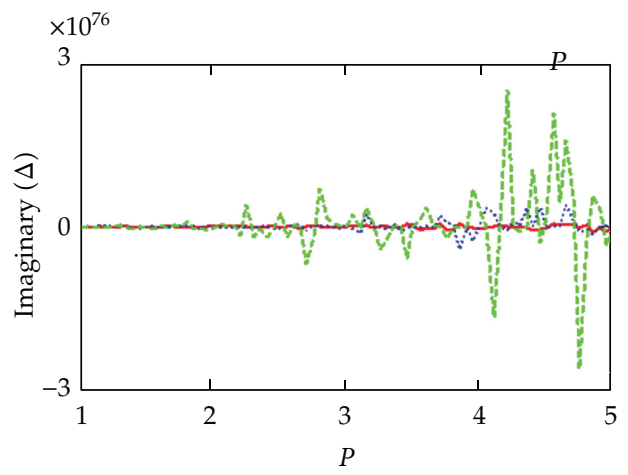

(b)

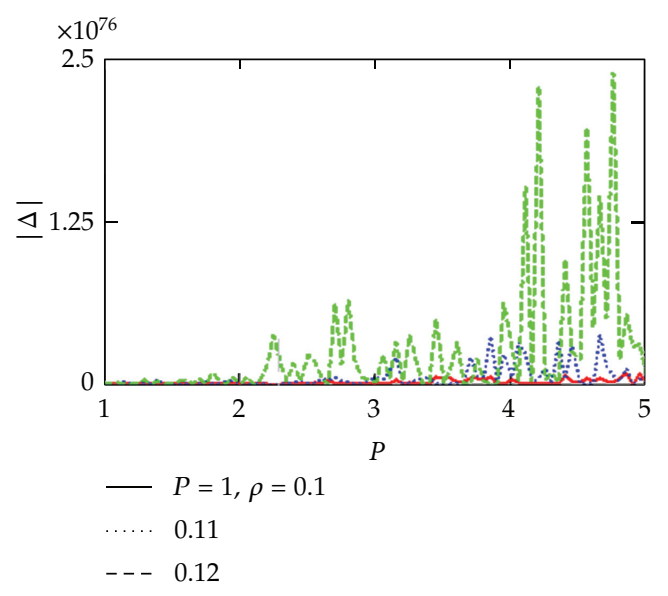

(c)

Figure 1: Effect of the density $\rho$ on (real, imaginary, and magnitude) of Stoneley waves determinant with respect to initial stress.

When the gravity field is neglected, there is no coupling between the constants $A_{j}, B_{j}$, and $E_{j}, F_{j}(j=3,4,5,6)$, the equations (3.10), (3.11) become, respectively,

$$
\begin{gathered}
\left(\nabla^{2}-S \frac{\partial}{\partial t}\right)\left[\left(c_{55}-\frac{P}{2}\right) \frac{\partial^{2} \psi}{\partial x_{1}^{2}}+\left(c_{33}-c_{31}-c_{55}-\frac{P}{2}\right) \frac{\partial^{2} \psi}{\partial x_{3}^{2}}-\rho \frac{\partial^{2} \psi}{\partial t^{2}}\right]+F \nabla^{4}\left(\frac{\partial \psi}{\partial t}\right)=0 \\
\left(\nabla^{2}-\frac{1}{x} \frac{\partial}{\partial t}\right)\left[\left(c_{11}+P\right) \frac{\partial^{2} \varphi}{\partial x_{1}^{2}}+\left(c_{13}+2 c_{55}+P\right) \frac{\partial^{2} \varphi}{\partial x_{3}^{2}}-\rho \frac{\partial^{2} \varphi}{\partial t^{2}}\right]-v_{1} \varepsilon \nabla^{2}\left(\frac{\partial \phi}{\partial t}\right)=0 .
\end{gathered}
$$

Equation (6.1) are in agreement with the corresponding equations obtained by Ahmed

In the absence of initial stress and when there is no coupling between the temperature and strain fields, (4.7) takes the form as obtained by Oshima [2]. 


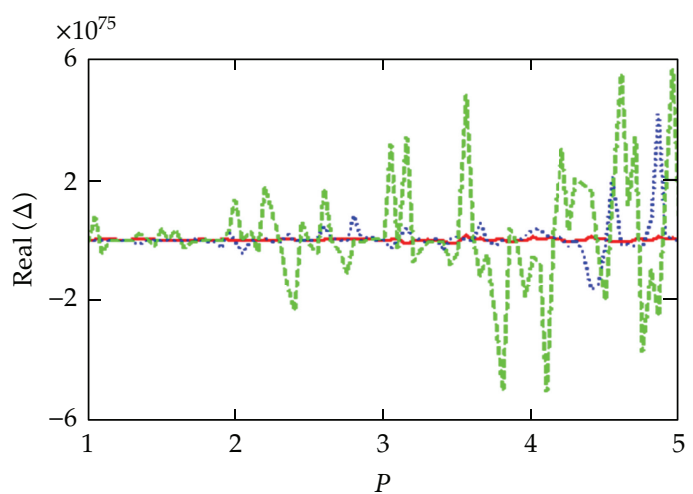

(a)

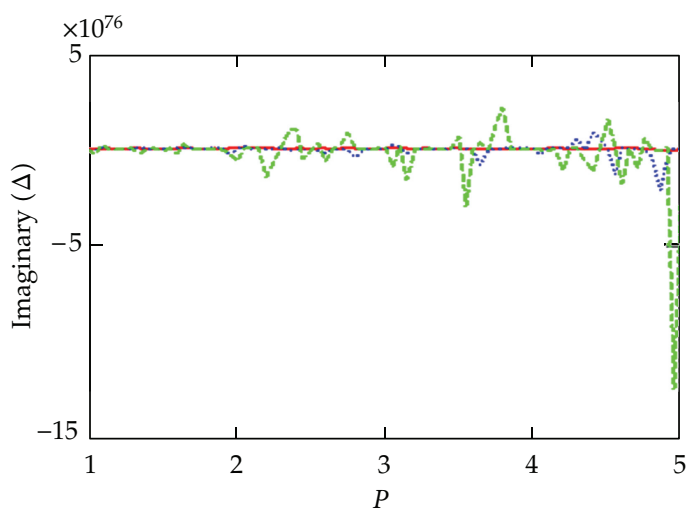

(b)

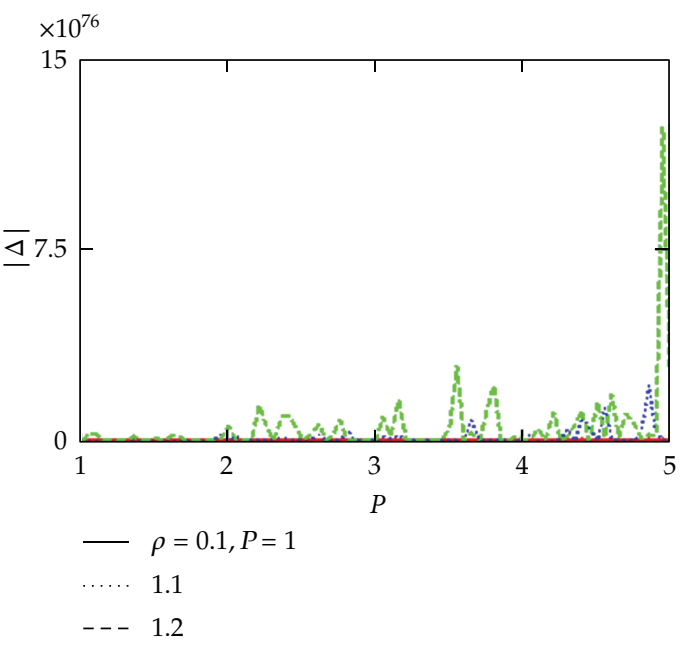

(c)

Figure 2: Effect of the parameter $b$ on (real, imaginary, and magnitude) of Stoneley waves determinant with respect to initial stress.

\section{Numerical Results and Discussions}

For a numerical computational work, Potash material is considered as an upper granular media with thickness $\mathrm{H}$ and $\mathrm{Fe}$ as lower granular media [28]

$$
\begin{array}{rrrrr}
\rho=1.098, & M=0.4, & F=0.5, & Q_{1}=0.4, & Q_{2}^{\prime}=0.6, \\
\rho^{\prime}=4.499, & M^{\prime}=0.6, & F^{\prime}=0.6, & Q_{1}^{\prime}=0.6, & Q_{2}^{\prime}=0.8 .
\end{array}
$$

A Mathcad program is used to invert the transform in order to obtain the results in the physical domain. From Figure 1, It is obvious that the determinant of Stoneley waves velocity increases and decreases with an increasing of the various values of the initial stress $P$, also with an increasing of the density $\rho$; the real and imaginary parts of the determinant of Stoneley waves frequency equation increase. It is seen that magnitude of determinant of the 
frequency equation for Stoneley waves takes a large decreasing with an increasing of the initial stress and density.

Figure 2 displays the influence of parameter $b$ on the frequency equation of Stoneley waves. It is concluded that the determinant of frequency equation of Stoneley waves increases with an increasing of the initial stress $P$. Also, it is clear that $\operatorname{Re}(\Delta)$ increases with an increasing of parameter $b$ but $\operatorname{Im}(\Delta)$ decreases.

Finally, it appears that the magnitude of $\Delta$ increases with the increased values of the parameter $b$.

\section{Conclusion}

From the results obtained, we concluded the following.

(1) The determinant of frequency of Rayleigh and Stoneley waves is affected by the influences of initial stress, density, gravity, and orthotropic of material and very pronounced on the waves propagation phenomena that indicates their utilitarian aspects in diverse fields as Geophysics, Geology, Acoustics, Plasma, and so forth.

(2) From Figures 1 and 2, it is obvious that the magnitude of Stoneley waves increases clearly with the influences of the density $\rho$ and parameter $b$.

(3) If the media considered are isotropic, the relevant results obtained deduce to the results obtained by El-Naggar [25].

\section{Nomenclature}

$c_{i j}$ : Are the elastic constants

$e_{i j}$ : Are the components of strain tensor

$F:$ Is the coefficient of fraction

$g:$ Is the acceleration due to the gravity

$M$ : Is the third elastic constant

$P: \quad$ Is the initial stress

$s:$ Is the specific heat per unit mass

$T:$ Is the temperature change about the initial temperature $T_{0}$

$v$ : Is the phase speed

$\alpha_{1}$ : Is the thermal expansion coefficient in the planes of orthotropic

$\alpha_{2}$ : Is the thermal expansion coefficient along the $x_{3}$-axis

$\rho:$ Is the density

$\tau:$ Is a function of depth

$\tau_{i j}$ : Are the components of stress tensor

$\omega:$ Is the frequency.

\section{References}

[1] G. Paria, "Bending of a shallow spherical shell under uniform pressures with the boundary partly clamped and partly simply-supported," Bulletin of the Calcutta Mathematical Society, vol. 52, pp. 79-86, 1960.

[2] N. Oshima, "A symmetrical stress tensor and its application to a granular medium," in Proceedings of the 3rd Japan National Congress for Applied Mechanics, vol. 77, pp. 77-83, 1955. 
[3] N. Oshima, "Dynamics of granular media," in Memoirs of the Unifying Study of the Basic Problems in Engineering Sciences by Means of Geometry, K. Kondo, Ed., vol. 2-3, 1955.

[4] R. K. Bhattacharyya, "Rayleigh waves in granular medium," Pure and Applied Geophysics PAGEOPH, vol. 62, no. 1, pp. 13-22, 1965.

[5] A. M. El-Naggar, "On the dynamical problem of a generalized thermoelastic granular infinite cylinder under initial stress," Astrophysics and Space Science, vol. 190, no. 2, pp. 177-190, 1992.

[6] S. M. Ahmed, "Rayleigh waves in a thermoelastic granular medium under initial stress," International Journal of Mathematics and Mathematical Sciences, vol. 23, no. 9, pp. 627-637, 2000.

[7] S. M. Ahmed, "Influence of gravity on the propagation of waves in granular medium," Applied Mathematics and Computation, vol. 101, no. 2-3, pp. 269-280, 1999.

[8] S. M. Ahmed, "Stoneley waves in a non-homogeneous orthotropic granular medium under the influence of gravity," International Journal of Mathematics and Mathematical Sciences, no. 19, pp. 31453155, 2005.

[9] A. M. Abd-Alla and S. M. Ahmed, "Rayleigh waves in an orthotropic thermo-elastic medium under gravity field and initial stress," Earth, Moon, and Planets, vol. 75, pp. 185-197, 1996.

[10] A. M. Abd-Alla and S. M. Ahmed, "Stoneley and Rayleigh waves in a non-homogeneous orthotropic elastic medium under the influence of gravity," Applied Mathematics and Computation, vol. 135, no. 1, pp. 187-200, 2003.

[11] A. M. El-Naggar, A. M. Abd-Alla, and S. M. Ahmed, "Rayleigh waves in a magnetoelastic initially stressed conducting medium with the gravity field," Bulletin of the Calcutta Mathematical Society, vol. 86, no. 3, pp. 243-248, 1994.

[12] S. C. Das, D. P. Acharya, and P. R. Sengupta, "Surface waves in an inhomogeneous elastic medium under the influence of gravity," Revue Roumaine des Sciences Techniques, vol. 37, no. 5, pp. 539-551, 1992.

[13] M. A. Goda, "The effect of inhomogeneity and anisotropy on Stoneley waves," Acta Mechanica, vol. 93, pp. 89-98, 1992.

[14] A. M. Abd-Alla, H. A. H. Hammad, and S. M. Abo-Dahab, "Rayleigh waves in a magnetoelastic halfspace of orthotropic material under influences of initial stress and gravity field," Applied Mathematics and Computation, vol. 154, no. 2, pp. 583-597, 2004.

[15] J. N. Sharma, V. Walia, and S. K Gupta, "Effect of rotation and thermal relaxation on Rayleigh waves in piezothermoelastic half space," International Journal of Mechanical Sciences, vol. 50, no. 3, pp. 433-444, 2008.

[16] J. N. Sharma, M. Pal, and D. Chand, "Propagation characteristics of Rayleigh waves in transversely isotropic piezothermoelastic materials," Journal of Sound and Vibration, vol. 284, no. 1-2, pp. 227-248, 2005.

[17] J. N. Sharma and V. Walia, "Further investigations on Rayleigh waves in piezothermoelastic materials," Journal of Sound and Vibration, vol. 301, no. 1-2, pp. 189-206, 2007.

[18] T. C. T. Ting, "Surface waves in a rotating anisotropic elastic half-space," Wave Motion, vol. 40, no. 4, pp. 329-346, 2004.

[19] N. Abo-el-nour and S. M. Abo-dahab, "Rayleigh waves in magneto-thermo-viscoelastic solid with thermal relaxation times," Applied Mathematics and Computation, vol. 149, no. 3, pp. 861-877, 2004.

[20] S. M. Ahmed and S. M. Abo-Dahab, "Propagation of love waves in an orthotropic granular layer under initial stress overlying a semi-infinite granular medium," Journal of Vibration and Control, vol. 16, no. 12, pp. 1845-1858, 2010.

[21] P. C. Vinh and G. Seriani, "Explicit secular equations of Rayleigh waves in a non-homogeneous orthotropic elastic medium under the influence of gravity," Wave Motion, vol. 46, no. 7, pp. 427-434, 2009.

[22] P. C. Vinh and G. Seriani, "Explicit secular equations of Stoneley waves in a non-homogeneous orthotropic elastic medium under the influence of gravity," Applied Mathematics and Computation, vol. 215, no. 10, pp. 3515-3525, 2010.

[23] P. C. Vinh, "Explicit secular equations of Rayleigh waves in elastic media under the influence of gravity and initial stress," Applied Mathematics and Computation, vol. 215, no. 1, pp. 395-404, 2009.

[24] W.-C. Lo, "Propagation and attenuation of Rayleigh waves in a semi-infinite unsaturated poroelastic medium," Advances in Water Resources, vol. 31, no. 10, pp. 1399-1410, 2008.

[25] A. M. El-Naggar, "On the dynamical problem of a generalized thermoelastic granular infinite cylinder under initial stress," Astrophysics and Space Science, vol. 190, no. 2, pp. 177-190, 1992. 
[26] A. M. Abd-Alla, S. M. Abo-Dahab, and F. S. Bayones, "Rayleigh waves in generalized magnetothermo-viscoelastic granular medium under the influence of rotation, gravity field, and initial stress," Mathematical Problems in Engineering, vol. 2011, Article ID 763429, 47 pages, 2011.

[27] A. Britan and G. Ben-Dor, "Shock tube study of the dynamical behavior of granular materials," International Journal of Multiphase Flow, vol. 32, no. 5, pp. 623-642, 2006.

[28] P. M. Morse and H. Feshbach, Methods of Theoretical Physics, Part I, McGraw-Hill, New York, NY, USA, 1953. 


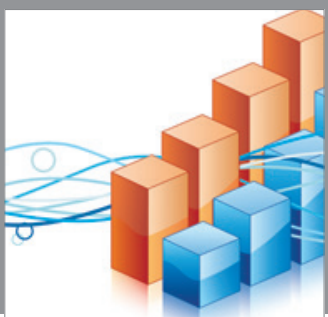

Advances in

Operations Research

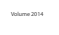

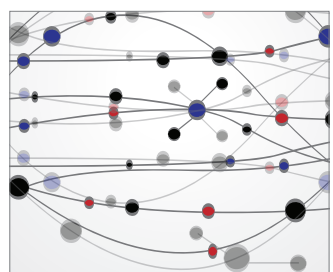

\section{The Scientific} World Journal
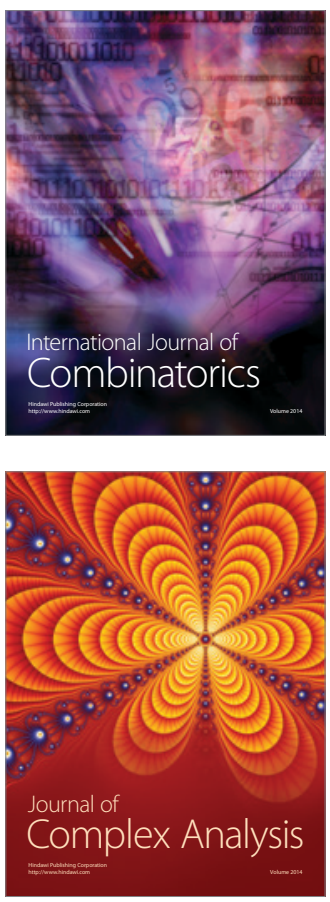

International Journal of

Mathematics and

Mathematical

Sciences
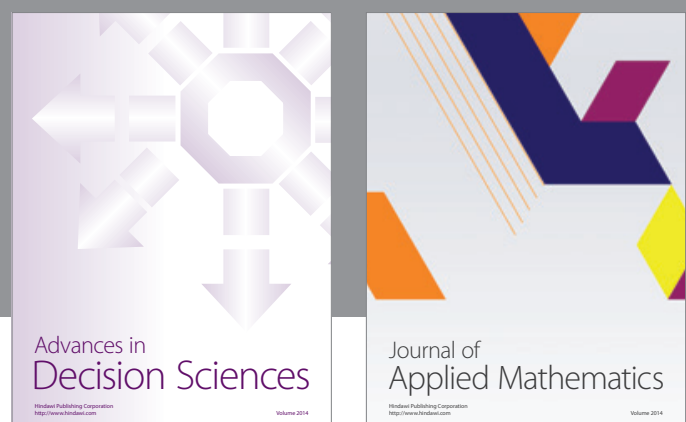

Journal of

Applied Mathematics
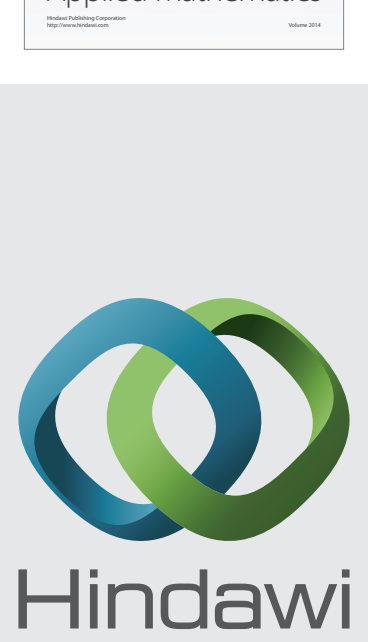

Submit your manuscripts at http://www.hindawi.com
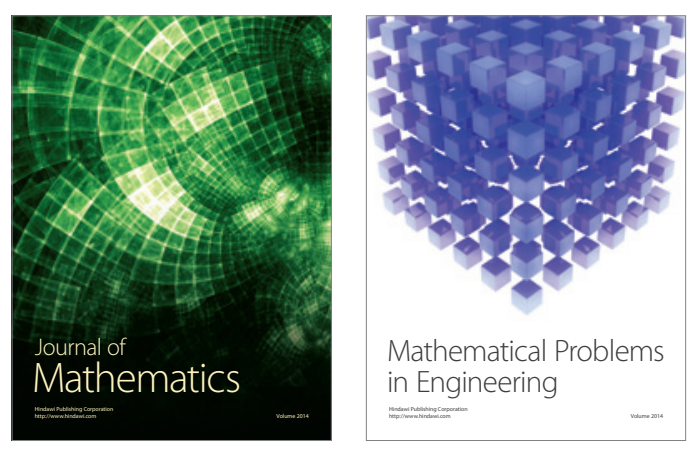

Mathematical Problems in Engineering
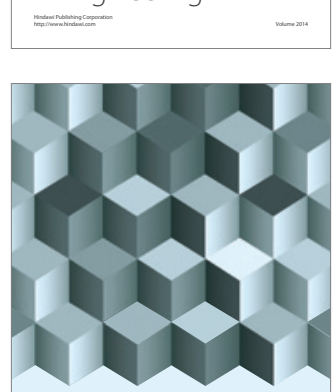

Journal of

Function Spaces
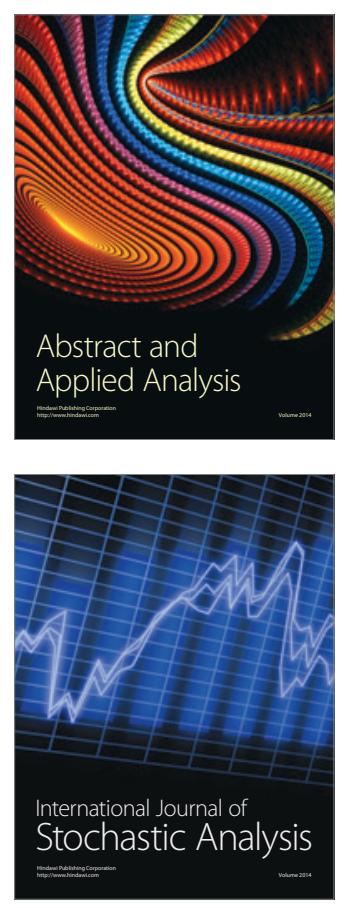

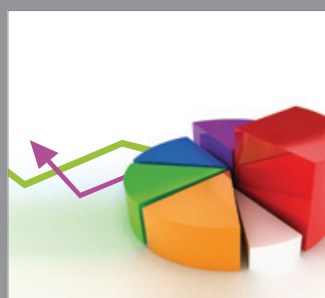

ournal of

Probability and Statistics

Promensencen
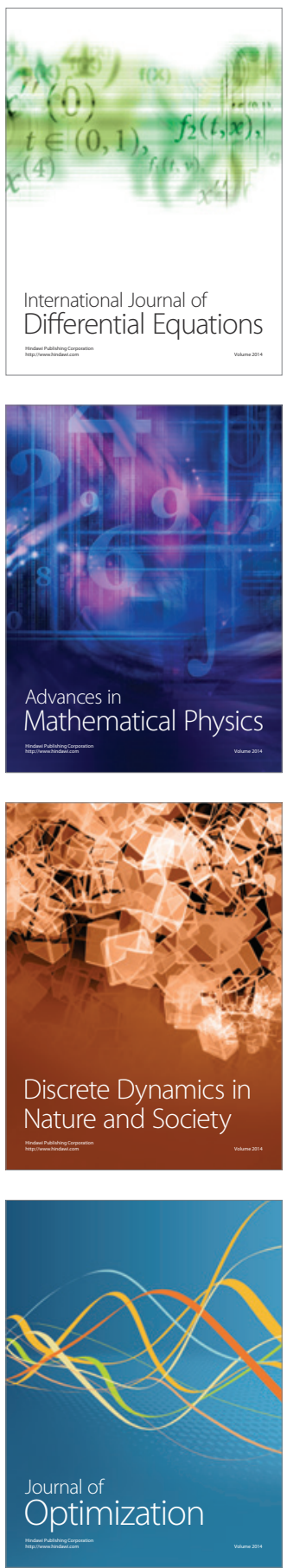